\title{
Potencial sinantrópico de mosquitos Kerteszia e Culex (D iptera: Culicidae) no Sudeste do Brasil
}

The synanthropic potential of Kerteszia and Culex mosquitoes (Diptera: Culicidae) in Southeastern Brazil

O swaldo Paulo Forattini, Iná Kakitani, Roseli La Corte dos Santos, Keilla Miki Kobayashi, Helene Mariko Ueno e Zoraida Fernández

Departamento de Epidemiologia da Faculdade de Saúde Pública da U niversidade de São Paulo. São Paulo, SP, Brasil 


\title{
Potencial sinantrópico de mosquitos Kerteszia e Culex (Diptera: Culicidae) no Sudeste do Brasil* \\ The synanthropic potential of Kerteszia and Culex mosquitoes (Diptera: Culicidae) in Southeastern Brazil
}

\author{
O swaldo Paulo Forattini, Iná Kakitani, Roseli La Corte dos Santos, Keilla Miki \\ Kobayashi, Helene Mariko U eno e Zoraida Fernández
}

Departamento de Epidemiologia da Faculdade de Saúde Pública da U niversidade de São Paulo. São Paulo, SP, Brasil

\section{Descritores}

Ecologia de vetores ${ }^{\#}$. Anopheles ${ }^{\#}$. Culex" Habitat. - Anopheles bellator. An. cruzii. Culex quinquefasciatus. Cx. sacchettae. Sinantropia.

\section{Resumo}

\section{Objetivo}

Observar a potencialidade sinantrópica de Anopheles bellator e de An. cruzii em vilarejo rodeado por ambiente natural.

\section{Métodos}

As observações foram levadas a efeito na localidade de Pedrinhas, no Sudeste do Estado de São Paulo, Brasil, de outubro de 1996 a janeiro de 2000. A sinantropia, ou domiciliação, foi estimada pelo índice de Nuorteva (s) acrescido do fator intradomiciliar de Mihályi (d) e adaptado para culicídeos hematófagos.

\section{Resultados}

Utilizando procedimento baseado na atração pela isca humana, as duas espécies de Kerteszia mostraram frequiência conjunta positiva correspondente a s=35,7. Isso parece indicar a existência de certo grau de sinantropia por parte desses mosquitos. Assim também, a população de $C x$. sacchettae, correspondente à razão de sinantropia $\mathrm{sr}=12,8$, obtida pelo cálculo da relação entre os dados conseguidos na armadilha de Shannon. Quanto ao método de procura ativa pela aspiração ambiental, os resultados obtidos consistiram em valores negativos de $s$. Assim, corresponderam a -43,1 para An. bellator, -48,2 para An. cruzii e -3,0 para $C x$. sacchettae. Tais cifras foram relacionadas ao valor positivo de $\mathrm{s}=+93,8$ apresentado por $C x$. quinquefasciatus e considerado como equivalente $\mathrm{a}+100,00$.

Conclusões

O observado em relação a domicílios isolados rurais repetiu-se em conjunto de casas formando vila, também de caráter rural, e circundada por ambiente primitivo. Ou seja, esses mosquitos adultos tendem a procurar o ambiente antrópico na busca de repasto sangüíneo. Uma vez tendo-o conseguido, voltam para o ambiente de onde saíram. Todavia, revelou-se alguma tendência adaptativa revelada pelo encontro de formas imaturas de An. bellator em recipientes artificiais. Quanto a $C x$. sacchettae, a população local parece mostrar tendência para adaptação ao meio antrópico.
Correspondência/Correspondence to:

Oswaldo Paulo Forattini

Núcleo de Pesquisa Taxonômica e Sistemática em Entomologia Médica (NUPTEM)

Faculdade de Saúde Pública da Universidade de São Paulo

Av. Dr. Arnaldo, 715

01246-904 São Paulo, SP, Brasil

E-mail: opforati@usp.br.
*Subvencionado pela Fundação de Amparo à Pesquisa do Estado de São Paulo - Fapesp (Proc. temático 95/0381-4). Edição subvencionada pela Fapesp (Processo no 00/01601-8).

Recebido em 21/6/2000. Aprovado em 2/10/2000. 


\section{Keywords}

Ecology, vectors ${ }^{\#}$. Anopheles ${ }^{\#}$. Culex". Habitat. - Anopheles bellator. An. cruzii. Culex quinquefasciatus. Cx. sacchettae. Synanthropy.

\begin{abstract}
Objective

To determine the synanthropic potential of Anopheles bellator and An. cruzii in a village close to a wild environment. For comparative purposes, Culex quinquefasciatus and $\mathrm{Cx}$. sacchettae populations were also investigated.

Methods

From October 1996 to January 2000, vectors investigations were carried out in Pedrinhas village, Southeastern of S. Paulo State, Brazil, through systematic collections with human bait, air aspirations and Shannon traps. The synanthropic index was estimated using Nuorteva's indices plus the Mihályi's endophylic factor. Results

Attraction principle was $\mathrm{s}=35.7$ for both Kerteszia species at the peridomiciliary environment through human bait. Cx. sacchettae showed a sr ratio of 12.8 with a degree of synanthropy. However active search through the aspiration method yielded negative s values, such as -43.1 for An. bellator and -48.2 for An. cruzii. For Cx. sacchettae that value was -3.0. These values were calculated when +100.00 was given to $\mathrm{Cx}$. quinquefasciatus, which showed the highest synanthropic habits, corresponding to $\mathrm{s}=+93.8$.

Conclusions

The present data allow to conclude that what was observed until now for isolated rural dwellings is valid for small villages at the same conditions. This means that female Kerteszia adults tend to be in anthropic environment for blood seeking. After that, they return to the surrounding natural environment of the village. Regarding $\mathrm{Cx}$. Sacchettae, they seem to have an anthropic adaptation tendency.
\end{abstract}

\section{INTRO DU ÇÃO}

É conhecido o comportamento de anofelíneos Kerteszia em relação ao ambiente domiciliar. As fêmeas adultas freqüentam as habitações à procura de fontes para a hematofagia. No entanto, tendem a manter o hábito silvestre e voltam ao ambiente natural, em seguida à realização do repasto sangüíneo (Forattini et al., ${ }^{1}$ 1990; Guimarães et al., ${ }^{9} 2000$ ).

Até agora e de maneira geral, as observações relatadas foram feitas objetivando casas rurais isoladas, geralmente localizadas nas proximidades do meio primitivo representado por floresta pluvial rica em bromélias. Em vista disso, é de interesse verificar a frequiência desses mosquitos em relação a conjunto habitacional instalado nas proximidades ou mesmo rodeado pelo ecossistema natural. Também, pouco se sabe das atividades diurnas nesse tipo de região. Em área litorânea do Sudeste do Brasil tem-se verificado que Anopheles cruzii mostra acentuada exofagia, ao passo que An. bellator apresenta maior endofilia (Forattini et al., 4,6 1996, 1999). Assim sendo, planejaram-se e foram executadas coletas destinadas a verificar tal comportamento, não apenas em casas isoladas, mas em conjunto habitado e rodeado pelo meio natural.

Objetivando o estabelecimento de comparações, observou-se, paralelamente, o comportamento de
Culex quinquefasciatus, uma vez que se trata de população considerada como acentuadamente domiciliada. Ao mesmo tempo e com a mesma finalidade, procurou-se verificar a atitude de culicídeo co-genérico, representado por $C x$. sacchettae. Aparentemente, trata-se de mosquito que está em pleno processo de adaptação sinantrópica (Forattini et al., ${ }^{3}$ 1995).

\section{MÉTO DOS}

A área estudada corresponde à localidade de Pedrinhas, no Sudeste do Estado de São Paulo, descrita detalhadamente em publicação anterior, assim como a metodologia utilizada (Forattini et al., ${ }^{7}$ 2000).

A sinantropia traduz adaptação ao meio antrópico. Para observá-la, há de se proceder a estudos comparativos entre as várias populações, mediante amostras obtidas ao longo de séries de coletas contemporâneas. De maneira geral, tal comparabilidade irá estabelecer a relação entre o fenótipo comportamental e as características do ambiente humano. Neste sentido, pode-se mencionar as pesquisas levadas a efeito com populações de dípteros não-picadores, as quais foram transplantadas, no que couberam, para as de hematófagos. Assim sendo, nas presentes observações modificouse, visando a adaptar os índices de Nuorteva ${ }^{11}$ (1963), para os dados obtidos mediante métodos 
de coleta comparáveis (Forattini et al., ${ }^{2} 1993$ ). Estes foram divididos em duas categorias gerais: os que utilizam a atração, representados pela isca humana (IH) juntamente com as armadilhas de Shannon (Shan), e os que empregam a busca, ou seja, mediante a aspiração (Asp). Quanto a estas últimas, procurou-se levar em conta fator de freqüência aos domicílios (d), de acordo com o conceito de Mihályi ${ }^{10}$ (1967), estabelecendo o valor de um, se positivo, e de 0 (zero), se negativo.

O índice de sinantropia (s) foi calculado de acordo com a fórmula:

$$
s=\frac{2 a+b-2 c}{2}
$$

onde:

- $\mathrm{a}=$ percentual dos espécimens obtidos no conjunto das coletas;

- $\mathrm{b}=$ percentual dos espécimens capturados na isca humana (IH);

- $\mathrm{c}=$ percentual dos espécimens coletados na mata circunjacente (Shan A).

Utilizou-se a procura ativa de mosquitos em locais que pudessem servir-lhes de abrigo. Para tanto, empregou-se a aspiração, levada a efeito em obediência a cronograma e ritmos já descritos (Forattini e col., $\left.{ }^{7} 2000\right)$.

\section{RESULTADOS}

\section{Atração}

De acordo com o critério descrito, para as coletas diurnas o índice s foi calculado com os resultados seguintes:

- An. bellator 19,7

- An. cruzii 16,0

- Cx. sacchettae 13,1

Considerando-se os dois representantes de Kerteszia verifica-se que, em conjunto, apresentaram valor de $s$ correspondente a 35,7 , o que parece indicar a existência de certo grau de sinantropia diurna. Todavia, nesse período as médias horárias (mh) foram comparáveis, tanto na mata (Shan A) quanto na vila (Shan B), para o horário de $15 \mathrm{~h}-18 \mathrm{~h}$. Notou-se aumento concernente ao período das $17 \mathrm{~h}$ 20h (Shan C) (Tabela 1).

Como se pôde verificar, mediante o uso da isca humana (IH) foram coletadas 2.079 fêmeas adultas, como resultado global de 87 capturas, 29 das quais se prolongaram até às $20 \mathrm{~h}$. No caso das efetuadas com as armadilhas de Shannon (Shan), obtiveram- se 3.332 fêmeas e somente $20(0,6 \%)$ machos. Dessa maneira, somente aqueles se referem os dados apresentados na Tabela 1.

No que concerne às razões de sinantropia (sr), foram feitos os seguintes cálculos:

$$
\begin{aligned}
& \operatorname{sr}(1)=\frac{\mathrm{IH}}{\text { ShanB }+ \text { ShanC }} \\
& \operatorname{sr}(2)=\frac{\text { Shan } B+\text { ShanC }}{\text { Shan } A}
\end{aligned}
$$

Assim, foram obtidos os seguintes valores:
- An. bellator
$0,8(\mathrm{srl})$ e $6,8(\mathrm{sr} 2)$
- An. cruzii
$0,7(\mathrm{srl})$ e $7,0(\mathrm{sr} 2)$
- Cx. sacchettae
$0,5(\mathrm{srl})$ e $12,8(\mathrm{sr} 2)$

\section{Aspiração}

Mediante a busca ativa com o uso do processo de aspiração foram coletados 5.553 espécimens adultos, dos quais $2.297(41,4 \%)$ fêmeas e $3.256(58,6 \%)$ machos. Os dados relativos a cada espécie e à aspiração (Asp) utilizada estão expostos na Tabela 2.

Para a avaliação da sinantropia usou-se o índice s, modificado com o acréscimo de $\mathrm{d}$, de acordo com a expressão:

$$
s=\frac{2 a+b+d-2 c}{2}
$$

na qual se considerou:

- $\mathrm{a}=$ percentual de espécimens obtidos no conjunto das coletas;

- $\mathrm{b}=$ percentual dos exemplares coletados no ambiente domiciliar (AspC-P, AspC-I, IH-Asp);

- $\mathrm{c}=$ percentual dos espécimens capturados na mata (Shan A);

- $\mathrm{d}=$ fator $(1,0$ ou 0,0$)$ de ocorrência intradomiciliar (IH-Asp).

Assim procedendo obtiveram-se os seguintes valores:
- An. bellator $-40,4$
- An. cruzii $-45,2$
- Cx. quinquefasciatus 93,8
- Cx. sacchettae $\quad-2,8$

Tais resultados sugerem alto grau de sinantropia $(+93,8)$ de $C x$. quinquefasciatus. Corrigindo este valor para $+100,0$, por comparação, as demais teriam valores correspondentes a:

- An. bellator $-43,1$

- An. cruzii -48,2

- Cx. sacchettae $-3,0$ 
Tabela 1 - Resultados obtidos na coleta de adultos fêmeas em Pedrinhas. As médias de Williams' (Xw) foram calculadas a partir dos dados com o uso da isca humana e as médias horárias graças aos conseguidos nas armadilhas de Shannon (Shan).

\begin{tabular}{|c|c|c|c|c|c|c|c|c|c|c|c|c|c|c|c|c|c|}
\hline \multirow[t]{3}{*}{ Espécies } & \multicolumn{5}{|l|}{$\mathrm{IH}$} & \multicolumn{12}{|c|}{ Shan } \\
\hline & \multirow{2}{*}{\multicolumn{2}{|c|}{$\frac{7 h-18 h}{n X w \bullet 100}$}} & \multicolumn{2}{|c|}{ 18h-20h } & \multirow{2}{*}{$\begin{array}{l}\text { Total } \\
\mathrm{n}\end{array}$} & \multicolumn{2}{|c|}{ Shan A (mata) } & \multicolumn{4}{|c|}{ Shan B (vila) } & \multicolumn{3}{|c|}{ Shan C (vila) } & \multicolumn{2}{|c|}{ Total } & \multirow[b]{2}{*}{$\mathrm{mh}$} \\
\hline & & & $\mathrm{n}$ & $\overline{X w} \cdot 100$ & & $\mathrm{n}$ & $\%$ & $\mathrm{mh}$ & $\mathrm{n}$ & $\%$ & $\mathrm{mh}$ & $\mathrm{n}$ & $\%$ & $\mathrm{mh}$ & $\mathrm{n}$ & $\%$ & \\
\hline $\begin{array}{l}\text { An. bellator } \\
\text { An. cruzii }\end{array}$ & $\begin{array}{l}682 \\
545\end{array}$ & $\begin{array}{l}172 \\
143\end{array}$ & $\begin{array}{l}213 \\
299\end{array}$ & $\begin{array}{l}155 \\
193\end{array}$ & $\begin{array}{l}895 \\
844\end{array}$ & $\begin{array}{l}157 \\
175\end{array}$ & $\begin{array}{l}40,9 \\
45,6\end{array}$ & $\begin{array}{l}1,0 \\
1,1\end{array}$ & $\begin{array}{l}125 \\
201\end{array}$ & $\begin{array}{l}32,1 \\
51,5\end{array}$ & $\begin{array}{l}0,9 \\
1,4\end{array}$ & $\begin{array}{r}935 \\
1.023\end{array}$ & $\begin{array}{l}36,6 \\
40,0\end{array}$ & $\begin{array}{l}6,8 \\
7,4\end{array}$ & $\begin{array}{l}1.217 \\
1.399\end{array}$ & $\begin{array}{l}36,5 \\
42,0\end{array}$ & $\begin{array}{l}2,8 \\
3,2\end{array}$ \\
\hline Cx. quinquefasciatu & tus 22 & 10 & 4 & 9 & 26 & - & - & - & - & 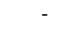 & - & - & - & - & - & - & - \\
\hline Cx. sacchettae & 94 & 41 & 220 & 137 & 314 & 52 & 13,5 & 0,3 & 64 & 16,4 & 0,4 & 600 & 23,4 & 4,3 & 716 & 21,5 & 1,6 \\
\hline Total & 1.343 & - & 736 & -2 & 2.079 & 384 & 100,0 & 2,5 & 390 & 100,0 & 2,7 & 2.558 & 100,0 & 18,5 & 3.332 & 100,0 & 7,6 \\
\hline
\end{tabular}

$\mathrm{n}=$ número de espécimens.

$\mathrm{IH}=\mathrm{isca}$ humana

$\mathrm{mh}=$ médias horárias

Shan=armadilha de Shannon

Tabela 2 - Número de espécimens coletados com as aspirações em Pedrinhas

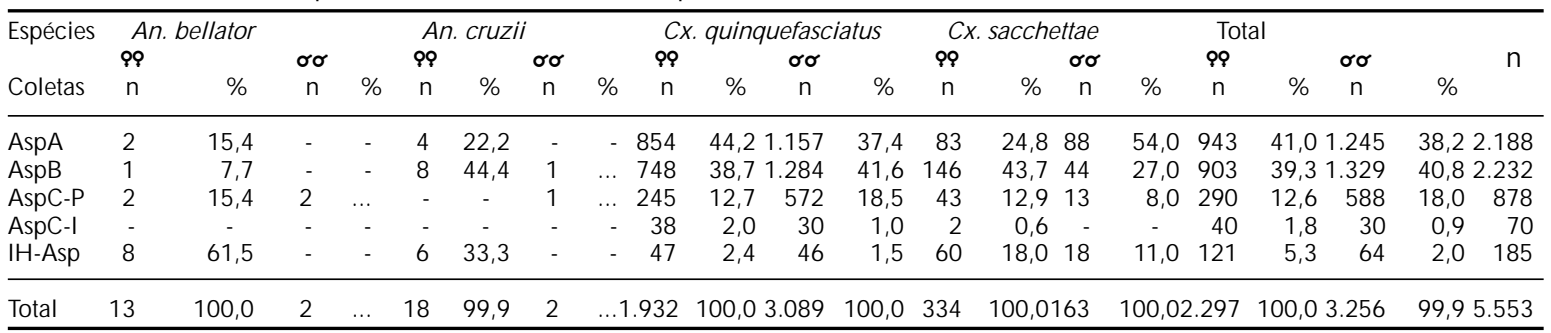

Asp=aspirações

\section{DISCUSSÃO}

Os resultados relatados permitem supor que as duas populações de Kerteszia freqüentam o ambiente antrópico à procura de realização do repasto sangüíneo, obedecendo a alguma inclinação antropófila. Porém, logo após realizá-lo, abandonam o ambiente à procura de abrigo no meio natural circundante. É bem verdade que os índices de sinantropia (s) a rigor foram calculados mediante dados obtidos por dois métodos distintos, ou seja, o da busca pela aspiração (Asp) e o da atração pelo uso da armadilha de Shannon (Shan A). Tal circunstância contra indicaria a comparação. Ocorre que o segundo desses métodos apresenta certo aspecto de ecletismo, o que torna a comparabilidade possível. Assim procedendo, pôde-se chegar a resultados tendendo a sugerir aquele comportamento.

Considerando as respostas genéticas de ordem adaptativa aos desafios do ambiente, há de se levar em conta a recombinação sexual. Por sua vez, esta varia muito e os processos dessa variabilidade continuam pouco conhecidos. É possível estabelecer conceito de "sistema adaptativo" para toda população que satisfaça os três postulados da seleção natural, ou seja, a existência de variabilidade e de continuidade propiciada pela herança e a ocorrência de diferenças nos êxitos (Frank, ${ }^{8}$ 1996). Assim sendo, encarando o comportamento das populações culicídeas focalizadas no que concerne a pequeno núcleo antrópico rural, ob- serva-se a atração exercida por este em relação àquelas. Esse fenômeno, observado na mesma região em domicílios rurais isolados, repetiu-se nessas circunstâncias, ou seja, no conjunto da vila (Forattini et al., ${ }^{4}$ 1996). Tanto adultos de An. bellator como de An. cruzii evidenciaram franca propensão a se dirigirem ao vilarejo à procura de fontes para a hematofagia. Os primeiros parecendo ter afinidade maior para sugar o homem (Forattini et al., ${ }^{6}$ 1999), embora após o repasto sangüíneo terem mostrado acentuada tendência à procura de abrigos no meio natural. Não obstante, o encontro de formas imaturas de An. bellator em recipientes artificiais da mesma localidade possa indicar início de desenvolvimento de processo adaptativo a esse ambiente antrópico (Forattini et al., ${ }^{5}$ 1998).

Quanto às espécies de Culex, como era de se esperar, a população de $C x$. quinquefasciatus mostrou elevado índice de domiciliação, a ponto dela ser tomada como paradigma. Assim, comparando-a com a de $C x$. sacchettae, esta mostrou-se bastante inferior. Contudo, em relação ao vilarejo, $C x$. sacchettae mostrou certa tendência adaptativa neste sentido, à semelhança do que já tinha sido observado em domicílio rural isolado (Forattini et al., ${ }^{3}$ 1995). Ao que parece, a população local de $C x$. sacchettae mostrou tendência mais acentuada à sinantropia diurna nesse período.

Em resumo, o que se tem observado a respeito do comportamento de mosquitos adultos em relação a domicílios rurais isolados, repete-se quando se trata 
de pequenos conjuntos de habitações em forma de vilas de caráter ainda rural, propiciado pela proximidade do meio primitivo circundante. É de se supor que, à medida que se dê o afastamento, em decorrência da atividade humana, o comportamento desses mosquitos potencialmente vetores sofrerá alteração.

\section{REFERÊNCIAS}

1. Forattini OP, Gomes A de C, Santos JLF, Kakitani I, $M$ arucci $D$. Freqüência ao ambiente humano e dispersão de mosquitos Culicidae em área adjacente à mata atlântica primitiva da planície. Rev Saúde Pública 1990;24:101-7.

2. Forattini OP, Kakitani I, Massad E, M arucci D. Studies on mosquitoes (Diptera: Culicidae) and anthropic environment. 3 - Survey of adult stages in rice irrigation system and the emergence of Anopheles albitarsis in South-Eastern Brazil. Rev Saúde Pública 1993;27:313-25.

3. Forattini OP, Sallum MAM, Kakitani I, Massad E, Marucci D. Studies on mosquitoes (Diptera: Culicidae) and anthropic environment. 8 - Survey of adult behaviour of Spissipes Section species of Culex (Melanoconion) in South-Eastern Brazil. Rev Saúde Pública 1995;29:100-7.

4. Forattini OP, Kakitani I, Massad E, M arucci D. Studies on mosquitoes (Diptera: Culicidae) and anthropic environment. 11 - Biting activity and blood-seeking parity of Anopheles (Kerteszia) in South-Eastern Brazil. Rev Saúde Pública 1996;30:107-14.

5. Forattini OP, Kakitani I, Marques GRAM, Brito M de. Formas imaturas de anofelíneos em recipientes artificiais. Rev Saúde Pública 1998;32:189-91.
Alguns, como $C x$. sacchettae aparentemente terão maior facilidade no caminho adaptativo. Outros, como os dois representantes de Kerteszia, mais dificilmente enveredarão por esse processo. Em virtude de sua baixa sinantropia, encontrarão maiores dificuldades à adaptação ao meio antrópico.
6. Forattini O P, Kakitani I, Santos RLC dos, U eno HM, Kobayashi KM. Role of Anopheles (Kerteszia) bellator as malaria vector in South-Eastern Brazil (Diptera: Culicidae). Mem Inst O swaldo Cruz 1999;94:715-8.

7. Forattini OP, Kakitani I, Santos RLC dos, Kobayashi KM, U eno HM, Fernandez Z. Comportamento de Aedes albopictus e de Ae. scapularis adultos (Diptera: Culicidae) no sudeste do Brasil. Rev Saúde Pública 2000;34:461-7.

8. Frank SA. The design of natural and artificial adaptive systems. In: Rose MR, Lauder GV, editors. Adaptation. San Diego: Academic Press; 1996. p. 451-505.

9. Guimarães $A E$, Gentile C, Lopes CM, Mello RP de. Ecology of mosquitoes (Diptera: Culicidae) in areas of Serra do Mar State Park, State of S. Paulo, Brazil. II $\mathrm{H}$ abitat distribution. Mem Inst $\mathrm{O}$ swaldo Cruz 2000; 95:17-28.

10. Mihályi $F$. The danger-index of the synanthropic flies. Acta Zool Acad Sci Hung 1967;13:373-7.

11. Nuorteva P. Synanthropy of blowflies (Dipt., Calliphoridae) in Finland. Ann Entomol Fenn 1963;29:1-49. 